\title{
Performance of a hybrid bistable device using an acoustooptic modulator
}

\author{
Ting-Chung Poon and Siu Kwan Cheung
}

\begin{abstract}
Previous studies on acoustooptic hybrid bistable devices have been limited to the Bragg regime involving two diffracted orders, and no comparisons have been made between experimental results and theoretical predictions. In this paper, a model including both acoustooptic diffraction and a nonlinear feedback path is investigated. The Klein-Cook parameter $Q$ has been brought into the investigation in that theoretical simulation results based on diffraction involving four diffracted orders are obtained. Experimental results are then presented and compared to theoretical predictions.
\end{abstract}

\section{Introduction}

Bistable optical devices have received much attention in recent years because of their potential applications in optical signal processing. 1,2 Nonlinearity and feedback are required to achieve bistability. A commonly used configuration is an etalon,,34 such as a Fabry-Perot cavity containing a nonlinear medium whose optical absorption is a function of the incident optical intensity. Feedback is possible as part of the incident intensity is transmitted or reflected to form an output intensity. Such a device which employs a nonlinear optical medium placed inside the cavity is an all-optical type, whereas one in which an artificial nonlinearity is created by using an electrooptic or acoustooptic switch and electrical feedback from a photodetector is known as a hybrid type. ${ }^{5-7}$ Although many all-optical devices have nonlinearities with very fast response times, they require high light intensities due to the inherently weak nonlinear optical effects. Thus they seem to be less well adapted to optical integration than the hybrid types, in which a relatively low optical input power is required to achieve bistability. However, the response times of the hybrid types are limited by the electronic circuitry. In this paper, the performance of a hybrid bistable device using an acoustooptic modulator is investigated.

The authors are with Virginia Polytechnic Institute \& State University, Bradley Department of Electrical Engineering, Blacksburg, Virginia 24061.

Received 4 October 1988.

0003-6935/89/224787-05\$02.00/0.

(C) 1989 Optical Society of America.

\section{Hybrid Bistable Optical (HBO) System}

The hybrid optical system under study basically consists of an acoustooptic modulator (AOM), a photodetector (PD), an electrical summer, and a feedback amplifier with gain $\beta_{0}$ (which includes the photodetector conversion efficiency) as shown in Fig. 1. The AOM is operated in the Bragg regime. ${ }^{8}$ The light diffracted into the first order is detected by the photodetector, amplified, summed with a bias voltage $V_{b}$, and fed back to the acoustic transducer to change the amplitude of its drive signal $V_{a}$, which in turn amplitude modulates (AM) the intensities of the diffracted light. Hence the feedback signal has a recursive influence on the diffracted light intensities.

It is well known that the relationship between the first-order light intensity $I_{1}$ and the rf driving voltage to the acoustooptic modulator is nonlinear. Therefore, the system has nonlinear feedback. Part of the signal processing in the system is through an electronic feedback circuit, while the rest is through an acoustooptic interaction. Based on the structure of this system, a mathematical model which includes both the acoustooptic interaction and a nonlinear feedback equation in terms of the parameters $\beta_{0}, V_{b}, V_{a}$, and $I_{1}$ can be constructed.

A plane wave theory $y^{9,10}$ is used to model the dynamics of the acoustooptic interaction process. The theory may be summarized in terms of the following set of coupled-differential equations:

$$
\begin{aligned}
\frac{d E_{n}}{d \xi}= & -j \frac{\alpha}{2}\left(\exp \left\{\frac{-j}{2} Q \xi\left[\frac{\phi_{\text {inc }}}{\phi_{\mathrm{B}}}+(2 n-1)\right]\right\} E_{n-1}\right) \\
& -j \frac{\alpha}{2}\left(\exp \left\{\frac{j}{2} Q \xi\left[\frac{\phi_{\text {inc }}}{\phi_{\mathrm{B}}}+(2 n+1)\right]\right\} E_{n+1}\right),
\end{aligned}
$$

with the boundary conditions $E_{n}=E_{\text {inc }} \delta_{n 0}$ at $z \leq 0$, 


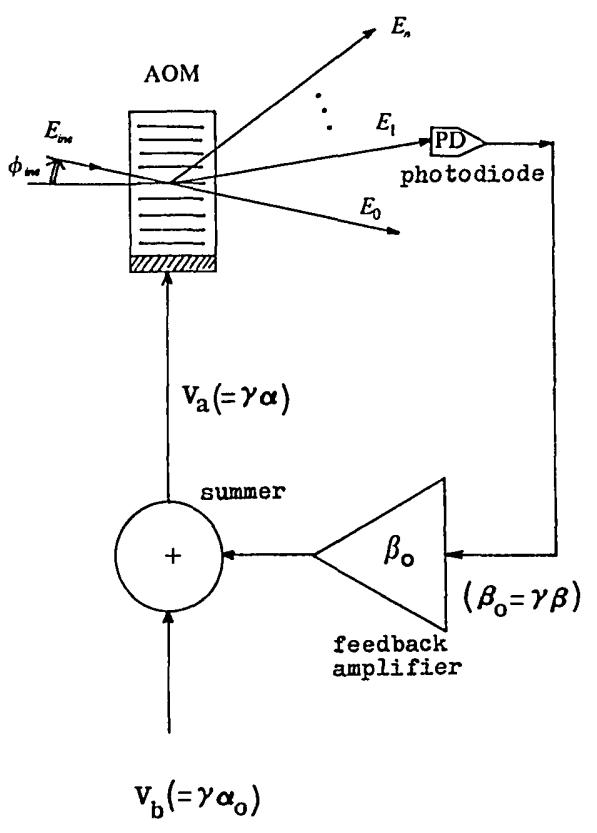

Fig. 1. Basic HBO system

where $\delta_{n 0}$ is the Kronecker delta, and $E_{n}$ is the complex amplitude of the $n$ th-order plane wave of light in the direction $\phi_{n}=\phi_{\text {inc }}+2 n \phi_{\mathrm{B}}$, where $\phi_{\text {inc }}$ is the incident angle of the plane wave $E_{\text {inc, }}$, and $\phi_{\mathrm{B}}$ is the Bragg angle. The sign convention for the angle is counterclockwise positive. The symbols in Eq. (1) are further defined as follows: $\alpha$ is the peak phase delay through the medium (proportional to the acoustic drive signal $V_{a}$ defined in Fig. 1), given by $\alpha=C k S L / 2$, where $C$ denotes a strain-optic coefficient of the medium, $k$ is the propagation constant of the light in the medium, $S$ is the amplitude of the sound field, and $L$ is the interaction length (i.e., the width of the sound column). $Q$ is the Klein-Cook parameter, ${ }^{10}$ which is given by $Q=K^{2} L / k$ with $K$ denoting the propagation constant of the sound. Finally, $\xi=z / L$ is a normalized position in the sound cell. At the exit of the sound column, $\xi=1$. The physical interpretation of Eq. (1) is obvious. The $n$ th-order light $E_{n}$ is generated from the scattering of the lower order $E_{n-1}$ and that of the higher-order $E_{n+1}$.

If now, the first-order diffracted light is detected by the photodetector and the resulting electrical signal amplified and summed with the external bias, and fed back to the acoustooptic modulator, as shown in Fig. 1, we have a system with nonlinear feedback. From Fig. 1 , the acoustic drive signal $V_{a}$ may be expressed as

$$
V_{a}=V_{b}+\beta_{0}\left|E_{1}\right|^{2} .
$$

Next, substituting $V_{a}=\gamma \alpha$, where $\gamma$ is a constant of proportionality relating the acoustic peak phase delay to an equivalent electric signal, we may rewrite Eq. (2) as

$$
\alpha=\alpha_{0}+\beta\left|E_{1}\right|^{2},
$$

where $\alpha$ is the peak phase delay scattering the light as defined before, $\alpha_{0}\left(=V_{b} / \gamma\right)$ and $\beta\left(=\beta_{0} / \gamma\right)$ are parame- ters proportional to the bias voltage $V_{b}$ and the gain of the amplifier $\beta_{0}$ described earlier, respectively.

The steady state behavior of the system is given by the simultaneous solution of Eqs. (1) and (3). Note, however, that Eq. (1) is an infinite set of coupled differential equations. We shall restrict ourselves to the case of four diffraction orders by choosing the nearest neighboring orders around $E_{0}$ and $E_{1}$; hence, i.e., $-1 \leq n \leq 2$. Thus for a plane wave incident at $\phi$ inc $=-\phi_{\mathrm{B}}$, Eq. (1) becomes

$$
\begin{aligned}
& n=-1: \frac{d E_{-1}}{d \xi}=-j \frac{\alpha}{2}[\exp (-j Q \xi)] E_{0}, \\
& n=0: \frac{d E_{0}}{d \xi}=-j \frac{\alpha}{2}\left[\exp (j Q \xi) E_{-1}+E_{1}\right], \\
& n=1: \frac{d E_{1}}{d \xi}=-j \frac{\alpha}{2}\left[E_{0}+\exp (j Q \xi) E_{2}\right], \\
& n=2: \frac{d E_{2}}{d \xi}=-j \frac{\alpha}{2}[\exp (-j Q \xi)] E_{1} .
\end{aligned}
$$

We study the behavior of the system based on Eqs. (3) and (4). Note that as $Q \rightarrow \infty$, i.e., in the pure Bragg regime, Eq. (4) becomes

$$
\begin{aligned}
& n=0: \frac{d E_{0}}{d \xi}=-j \frac{\alpha}{2} E_{1}, \\
& n=1: \frac{d E_{1}}{d \xi}=-j \frac{\alpha}{2} E_{0},
\end{aligned}
$$

and the solutions

$$
\begin{aligned}
& E_{0}=E_{\text {inc }} \cos (\alpha \xi / 2), \\
& E_{1}=E_{\text {inc }}(-j) \sin (\alpha \xi / 2)
\end{aligned}
$$

are the well-known expressions for the scattered light in Bragg diffraction. Therefore, the nonlinearity involving two diffracted orders is a sine squared function.

\section{Simulations}

In the simulations the effective $\alpha$ is treated as constant, i.e., the sound pressure remains constant during interaction with light. This is true if and only if the acoustooptic interaction time, given as the ratio of the laser beamwidth and the bulk speed of sound in the acoustooptic modulator, is much smaller compared to the delays incorporated by the finite response time of the photodetector, the rf modulator drive, and the feedback amplifier or any other delay that may be installed (e.g., a coaxial cable) in the feedback path. Now, for fixed values of $\alpha_{0}$ and $\beta$, the output $E_{1}$ will undergo a series of iterations at every instant $\alpha$ is updated through the feedback action. A discrete time method is employed to analyze the continuous time system. Let $E_{1}(m)$ be the intermediate solution of Eqs. (3) and (4) after $m$ iterations, starting from initial conditions $E_{0}(0)=1$ and $E_{1}(0)=E_{2}(0)=E_{-1}(0)=0$. Since we are investigating the steady state behavior of the system, an equilibrium state must be imposed after some iterations. The following criterion is used to determine the equilibrium state:

$$
\left|E_{1}(m+1)-E_{1}(m)\right|<\epsilon,
$$



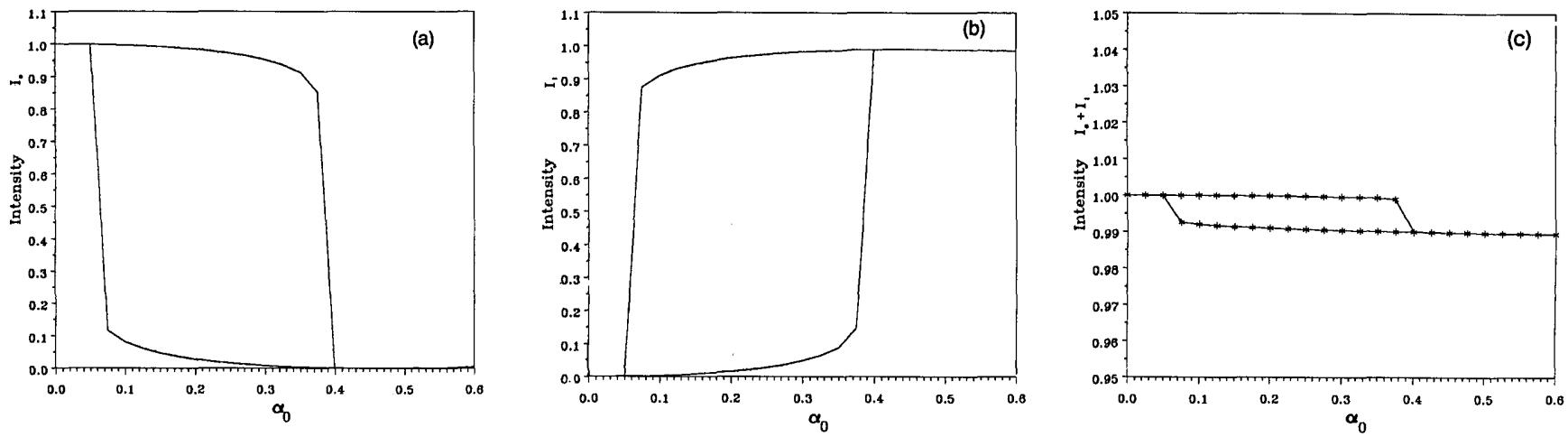

Fig. 2. Hysteresis curve (intensity vs bias, $\alpha_{0}$ ): $\quad \beta=2.6$ and $Q=20$ (a) for the zeroth-order light $I_{0}$; (b) for the first-order light $I_{1}$; and (c) for $I_{0}$ $+I_{1}$.

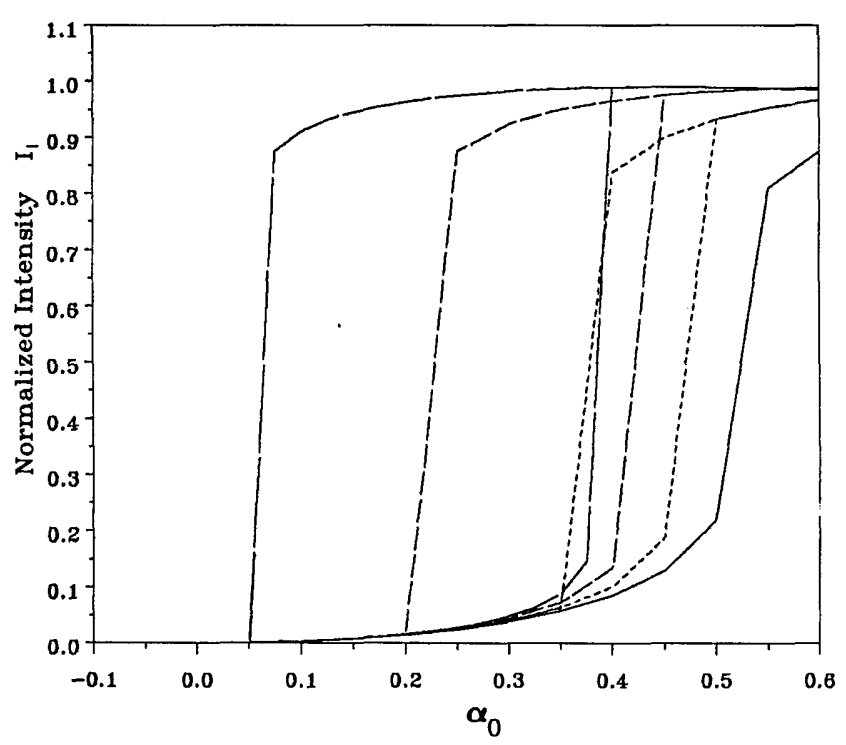

Legend:

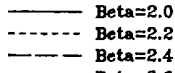

$($ Beta $=\beta)$

Fig. 3. Hystereses for first-order diffracted light with different feedback gains $\beta(Q=20)$.

where $\epsilon$ is the error tolerance placed on the difference between the two consecutive iterations $m$ and $m+1$. If $\left|E_{1}(m)\right|$ does not converge, we set a maximum limit of $m, m_{\max }=200$. In this way a set of $E_{1}(m)$ in the steady state can be obtained for different values of $\alpha_{0}$. Note that it is sufficient for a discrete time dynamic system to be unstable if its corresponding continuous-time dynamical system is unstable. However, an unstable discrete-time system does not necessarily imply an unstable continuous time system. ${ }^{11,12}$

In our simulations, the range of $\alpha_{0}$ is from 0 to 0.6 with an increment of $\delta \alpha_{0}$. The value of $\delta \alpha_{0}$ also determines the accuracy of the results. In all our simulations we take $\epsilon=10^{-5}$ and $\delta \alpha_{0}=0.05$ and employ a fifth-order Runge-Kutta numerical method to find the solution of the coupled difference equations. ${ }^{13}$ Figures 2(a) and (b) show the zeroth- and first-order diffracted light intensities, $I_{0}$ and $I_{1}$, respectively, as

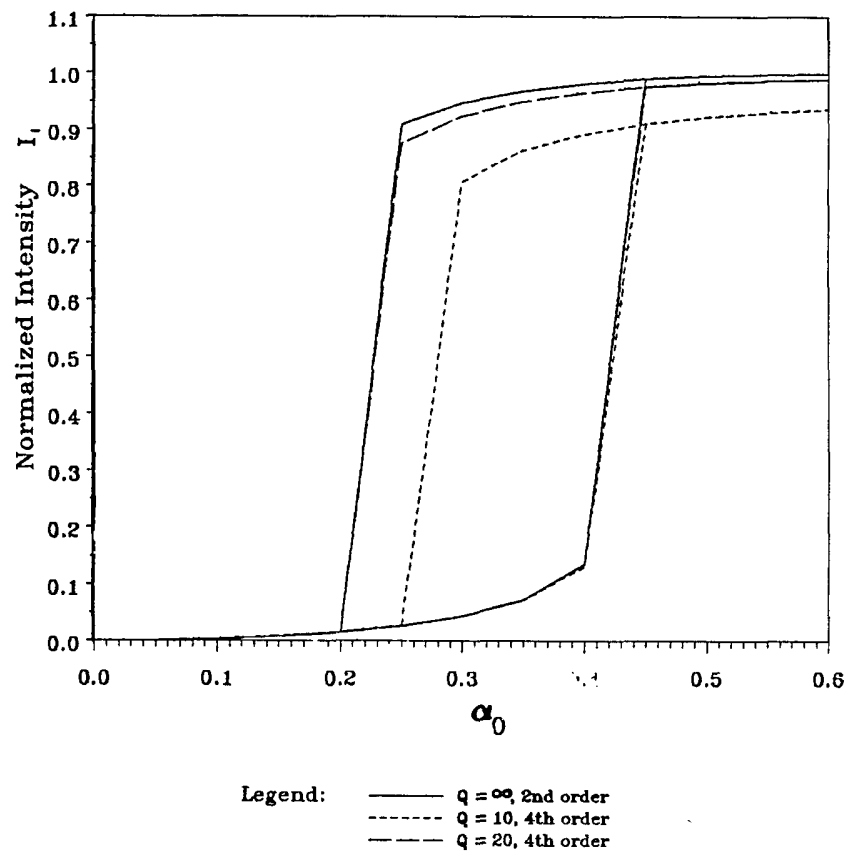

Fig. 4. Hystereses for first-order light as a function of $Q(\beta=2.4)$.

functions of bias $\alpha_{0}$. Figure 2(a) illustrates complementary bistable switching, ${ }^{14}$ and Fig. 2(c) shows the sum of the two intensities $I_{0}$ and $I_{1}$, and we observe that it also exhibits hysteretic behavior. Note that most of the energies are switched between the zeroth and first orders. Figure 3 shows the hysteresis curves for the first-order light by changing $\beta$ with a fixed $Q=20$. No hysteresis is observed when $\beta=2$, and the upper limit of $\beta$ is around 3.5. Beyond that value, the system becomes unstable. Note that the hysteresis curve moves to a lower bias region as the feedback gain increases. This feature may be important for some tuning purposes. Note also that the system shows a widening of the hysteresis loop more significant to the left with an increasing feedback gain. Finally, in the last simulation, Fig. 4 shows the performance of the system by changing $Q$ with a fixed $\beta=2.4$. It is observed that an increase of $Q$ will heighten and widen the hysteresis loop. Note that the case of $Q \rightarrow \infty$ corresponds to an ideal Bragg diffraction where only 


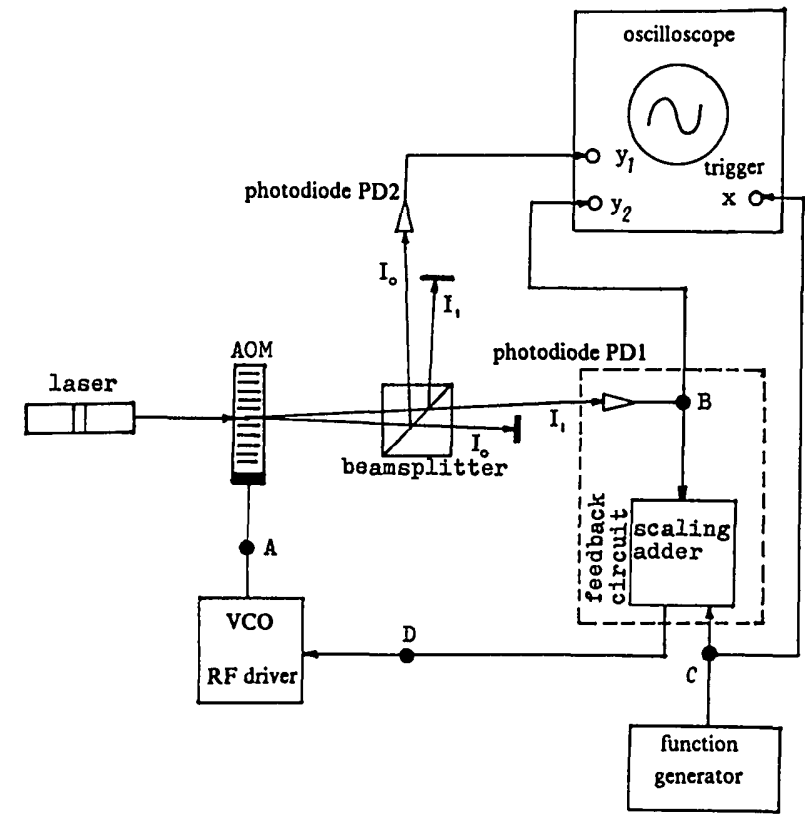

Fig. 5. Schematic diagram of the experimental setup.

two diffracted orders are involved. ${ }^{10}$ In the next section, we show some experimental results, which will confirm some of the observations made in this section.

\section{Experimental Results}

A schematic diagram of the experimental setup is shown in Fig. 5. An AOM-40 acoustooptic modulator $^{15}$ operates in the Bragg regime with $Q=20$. The center frequency of this modulator is at $40 \mathrm{MHz}$. For simplicity, only two diffracted orders are shown in the drawing. Diffraction into higher orders, however, is observable. The two diffracted orders are split into two separate paths. One path leads to photodetector $P D 1$ of the feedback circuit and the other to photodetector PD2. The feedback circuit includes a scaling adder and a photodiode detector circuit. To display the hysteretic behavior of the system, a triangular waveform is injected into the scaling adder at node $C$ (corresponding to $V_{b}=\gamma \alpha_{0}$ in Fig. 1) of the feedback circuit. The upper trace in Fig. 6 shows the triangular waveform, while the lower trace is the nonlinear feedback signal at node $D$. Note that in the feedback signal there are two sudden jumps corresponding to the two thresholds of the hysteresis loop for every cycle of occurrence.

Figures 7(a)-(c) show the hysteresis curves (intensity vs bias voltage $V_{b}$ ) as a function of increasing $\beta_{0}$. The upper and lower curves for each figure correspond to the zeroth orders and first orders, respectively. These experimental results confirm the theoretical prediction qualitatively. Specifically, the curves move to a lower bias voltage region, and the areas of the width of the loops widen as the feedback gain increases. Figure 8 shows a comparison of the experimental and simulation results. Note that the widths of both loops are approximately the same, and qualitative agreement with theory is satisfactory; but there

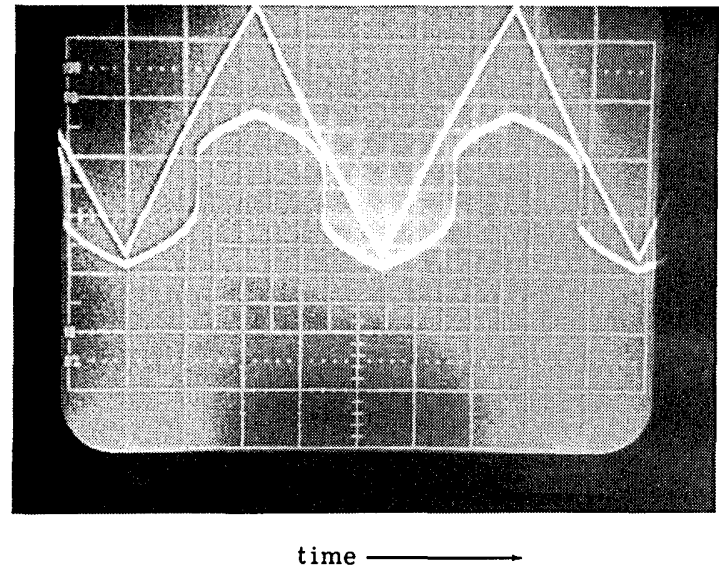

Fig. 6. Traces showing the bias voltage waveform (upper) and feedback signal waveform (lower) at nodes $C$ and $D$, respectively, in Fig. 5.

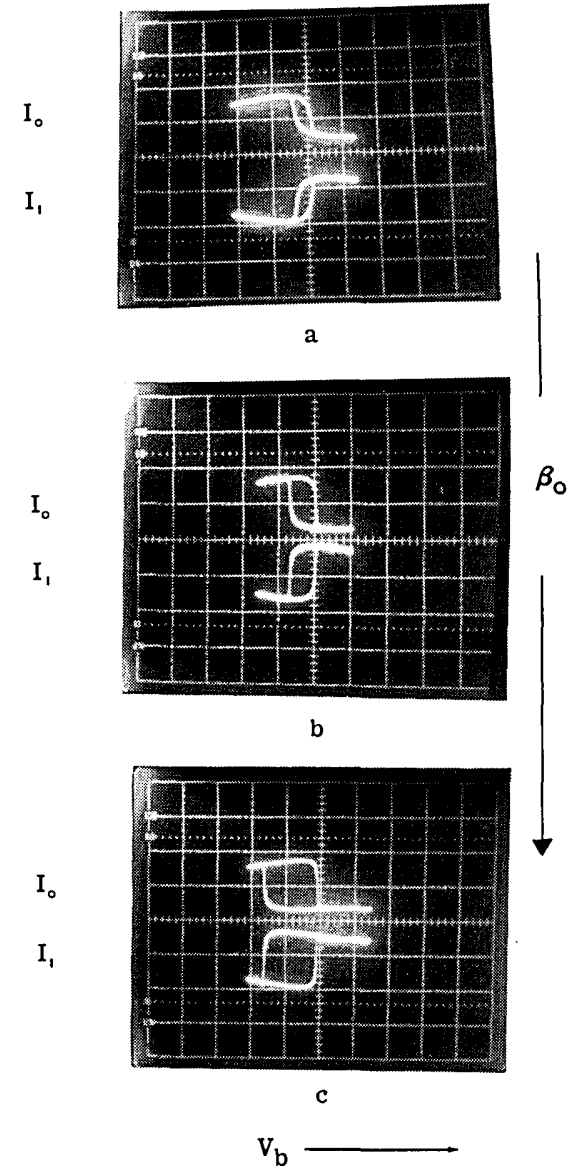

Fig. 7. Oscilloscope traces showing hysteresis curves as a function of increasing values of feedback gain $\beta_{0}(Q=20)$.

exist some quantitative differences. Possible reasons for the discrepancies are: (1) the nonlinearity of the electronic circuitry which may occur when feedback is employed; (2) the finite bandwidth of the electronic circuit (since the feedback circuit employs 741 operational amplifiers and is designed to have a maximum gain of 10 , the bandwidth of this circuit, therefore, does 


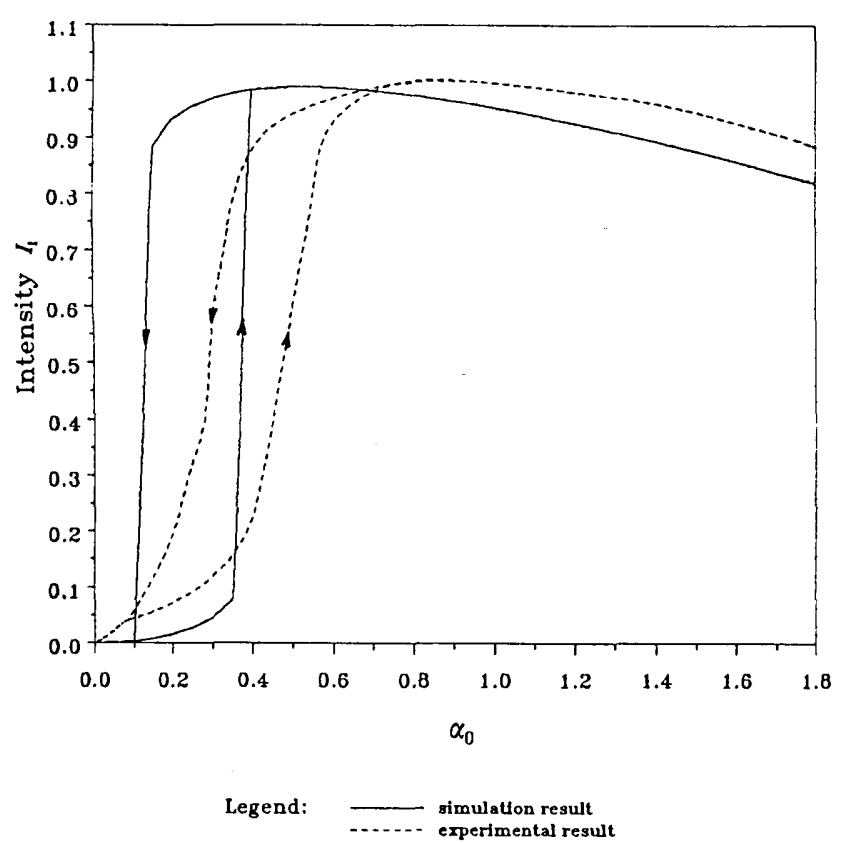

Fig. 8. Comparison between experimental and simulation results. The measured $\beta$ is 2.52 and $Q=20$.

not exceed $100 \mathrm{kHz}$ ); (3) the finite response time for the acoustooptic interaction which is neglected in the computer simulations; and (4) the numerical convergence criterion [see Eq. (7)] used in the simulation to determine the equilibrium state.

\section{Concluding Remarks}

The performance of a hybrid bistable system based on acoustooptic Bragg diffraction has been studied. Simulations show that the performance of the system is better with a larger feedback gain, which increases the width of the hysteresis curve. Also, results indicate that the hysteresis moves to a lower bias region for a larger feedback gain. These observations are confirmed by experimental results. We have also compared the simulation and experimental results for a particular value of the feedback gain $(\beta=2.52)$. Qualitative agreement between the two results is satisfactory. The performance of the device as a function of the Klein-Cook parameter $Q$ has also been investigated. Simulations show improved performance with a larger value of $Q$.
As a final note, it may be mentioned that in this paper we have considered diffraction in the first-Bragg regime, i.e., $\phi_{\text {inc }}=-\phi_{\mathrm{B}}$. The possibility of achieving bistability by operating the acoustooptic modulator in the higher Bragg regime (i.e., $\phi_{\text {inc }}=-m \phi_{\mathrm{B}}$, where $m$ is an integer greater than unity) also exists. In the latter case, the diffracted order fed back to the acoustooptic modulator is $I_{m}$ instead of $I_{1}$. Preliminary simulation results for the second Bragg regime $(m=2)$ indicate that hysteresis can be obtained for a much higher value of $\beta$ compared with that of the first Bragg regime. We plan to investigate this further in the future.

\section{References}

1. P. W. Smith and W. J. Tomlinson, "Bistable Optical Devices Promise Subpicosecond Switching," IEEE Spectrum 18, 26-33 (1981).

2. D. A. B. Miller, "Bistable Optical Devices: Physics and Operating Characteristics," Laser Focus 79-82 (Apr. 1982).

3. H. M. Gibbs, S. L. McCall, and T. N. C. Venkatesan, "Differential Gain and Bistability Using a Sodium-Filled Fabry-Perot Interferometer," Phys. Rev. Lett. 36, 1135-1138 (1976).

4. P. W. Smith and E. H. Turner, "Bistable Fabry-Perot Devices," IEEE J. Quantum Electron. QE-13, 42D (1977).

5. E. Garmire, J. H. Marburger, and S. D. Allen, "Incoherent Mirrorless Bistable Optical Devices," Appl. Phys. Lett. 32, 320 321 (1978).

6. J. Chrostowski and C. Delisle, "Bistable Optical Switching Based on Bragg Diffraction," Opt. Commun. 41, 71-74 (1982).

7. P. P. Banerjee and T.-C. Poon, "Simulation of Bistability and Chaos in Acousto-Optic Devices," in Proceedings, Midwest Symposium on Circuits and Systems (Elsevier, Amsterdam, 1987), pp. 820-823.

8. A. Korpel, Acousto-Optics (Marcel Dekker, New York, 1988).

9. A. Korpel and T.-C. Poon, "Explicit Formalism for AcoustoOptic Multiple Plane-Wave Scattering," J. Opt. Soc. Am. 70, 817-820 (1980).

10. T.-C. Poon and A. Korpel, "Feynman Diagram Approach to Acousto-Optic Scattering in the Near-Bragg Region," J. Opt. Soc. Am. 71, 1202-1208 (1981).

11. T. S. Parker and L. O. Chua, "Chaos: A Tutorial for Engineers," Proc. IEEE 75, 982-1007 (1987).

12. C. F. Gerald, Applied Numerical Analysis (Addison-Wesley, Reading, MA, 1978).

13. L. John and R. Reiss, Numerical Analysis (Addison-Wesley, Reading, MA, 1982).

14. S. Tarucha, M. Makoto, and J. Noda, "Complementary Optical Bistable Switching and Triode Operation Using $\mathrm{LiNbO}_{3}$ Directional Coupler," IEEE J. Quantum Electron. QE-17, 321-324 (1981).

15. Intra-Action Corp., 766 Foster Ave., Bensenville, IL 60106.

The authors would like to acknowledge discussions with P. P. Banerjee and G. Indebetouw. M. R. Chatterjee's input in this work is also greatly appreciated. 